XXII-3 | 2017

Forms of Activism in the United Kingdom (Grassroots Activism, Culture, Media)

\title{
Les Community arts en Grande-Bretagne : un mouvement artistique engagé, 1968-1990
}

Community Arts in Britain : a Committed Artistic Movement, 1968-1990

\section{Mathilde Bertrand}

\section{(2) OpenEdition}

\section{Journals}

Édition électronique

URL : http://journals.openedition.org/rfcb/1523

DOI : $10.4000 /$ rfcb.1523

ISSN : 2429-4373

Éditeur

CRECIB - Centre de recherche et d'études en civilisation britannique

Référence électronique

Mathilde Bertrand, "Les Community arts en Grande-Bretagne : un mouvement artistique engagé,

1968-1990 ", Revue Française de Civilisation Britannique [En ligne], XXII-3 | 2017, mis en ligne le 05 juillet 2017, consulté le 01 mai 2019. URL : http://journals.openedition.org/rfcb/1523 ; DOI : 10.4000/ rfcb. 1523

Ce document a été généré automatiquement le 1 mai 2019.

\section{(i) $\$$}

Revue française de civilisation britannique est mis à disposition selon les termes de la licence Creative Commons Attribution - Pas d'Utilisation Commerciale - Pas de Modification 4.0 International. 


\title{
Les Community arts en Grande- Bretagne : un mouvement artistique engagé, 1968-1990
}

\author{
Community Arts in Britain : a Committed Artistic Movement, 1968-1990
}

\author{
Mathilde Bertrand
}

\section{Introduction}

1 À la fin des années soixante, dans le sillage de la contre-culture, apparaissent à travers le Royaume-Uni des collectifs d'artistes issus de différentes disciplines (théâtre, photographie, peinture, sculpture, vidéo, musique) qui ouvrent des espaces dédiés aux pratiques artistiques dans des quartiers urbains pauvres et dépourvus de structures culturelles pouvant constituer un accès à l'art. Loin des schémas élitistes dominants, il ne s'agit pas tant de sortir l'art de l'establishment pour le faire venir aux classes populaires, que de créer les conditions d'émergence de pratiques et de formes culturelles issues de ces classes populaires, et dans des contextes qui garantissent aux acteurs un contrôle sur les usages de ce qu'ils produisent. Les pratiques community arts sont motivées par « une ambition politique de mettre les moyens de production artistique entre les mains des gens ${ }^{1}$.» Diverses et foisonnantes dans leur application, les pratiques community arts se propagent en réseau à partir du milieu des années soixante-dix, notamment grâce à la création de l' Association of Community Artists en 1974. Les projets community arts naissent de l'engagement d'artistes dans les communautés, mais aussi, dans certains cas, de la mobilisation des membres d'une communauté, sans formation spécifique dans le domaine de l'art, et qui initient localement des actions collectives au sein desquelles la créativité artistique occupe une place essentielle. Ces projets artistiques et culturels se conçoivent comme des mobilisations collectives à part entière, qui permettent à la population d'un quartier, d'une localité, d'interpeller les pouvoirs publics, et plus largement de participer à la prise de décision, ainsi qu'aux débats politiques à l'échelle locale de la communauté. 
2 Les sociologues définissent un mouvement social comme une mobilisation porteuse d'un projet alternatif ${ }^{2}$, une "action collective concertée en faveur d'une cause " ${ }^{3}$, portée par des acteurs qui s'y engagent volontairement, en vue de la réalisation d'objectifs politiques. Les pratiques community arts, dans les formulations qu'elles se donnent, les projets qu'elles mettent en œuvre, et l'engagement des acteurs, renvoient à ces caractéristiques. La revendication d'un accès démocratique à la production artistique est indissociable d'un objectif politique d'autonomisation des individus et des groupes sociaux marginalisés. Les pratiques community arts, vecteurs d'expressions culturelles populaires, représentent non seulement une remise en question fondamentale des conceptions hiérarchiques de la culture, mais sont également porteuses d'un projet social où se mêlent étroitement production artistique et culturelle et engagement des acteurs dans la vie locale.

3 La notion d'engagement fournit un prisme d'analyse pertinent pour tenter de cerner les objectifs et caractéristiques de ce mouvement artistique inscrit dans des luttes politiques. Quels sont les thèmes par rapports auxquels il se structure? Quelles sont les facteurs de mobilisation des acteurs qui initient des projets community arts, et les formes d'engagement des participants qui s'y associent? Profondément influencé par les théoriciens de l'émancipation ${ }^{4}$, le mouvement conçoit ses objectifs en termes d'autonomisation des individus et des groupes, dans des démarches d'empowerment: comment l'articulation entre accès aux pratiques artistiques et participation politique est-elle envisagée ? Enfin, si le mouvement se définit au cours des années soixante-dix selon une éthique de l'action collective, qu'advient-il de son élan radical dans le contexte idéologique de la décennie suivante, marquée par l'ascendance de valeurs individualistes et conservatrices?

\section{Friches artistiques et quartiers défavorisés : des réponses par l'art}

4 Les projets community arts qui apparaissent de manière spontanée à partir de la fin des années soixante partent du même constat: les possibilités d'expression de la culture populaire sont très fortement réduites en raison du manque criant d'espaces dédiés aux activités artistiques ou de loisirs dans les nouveaux lotissements construits dans l'aprèsguerre. Les plans d'urbanisme des années cinquante et soixante développés dans le contexte de programmes de rénovation urbaine de grande ampleur n'ont pas fait de la culture et des espaces de sociabilité leur priorité. Les urbanistes et les autorités locales ont privilégié la construction de tours d'immeubles plutôt que de rénover l'habitat des quartiers ouvriers anciens, plus intégrés ${ }^{5}$. Les relations traditionnelles de solidarité au sein du voisinage dans les quartiers ouvriers s'en trouvent profondément transformées. Dans les nouveaux quartiers, l'enfance et la jeunesse, les femmes sans emploi rémunéré et les personnes âgées ou isolées ne disposent pas de locaux ou de ressources matérielles propices à la sociabilité, et la verticalité des tours d'immeuble ne facilite pas les rapports de voisinage. Dès 1957, Michael Young et Peter Wilmott, sociologues des sociabilités urbaines, décrivent ces transformations et soulignent le phénomène d'appauvrissement culturel dans les nouveaux quartiers de logements sociaux, où les activités de loisirs se recentrent sur la famille nucléaire au détriment des relations de voisinage ${ }^{6}$. 
5 C'est dans ce contexte qu'apparaissent à partir de 1969 les premières initiatives artistiques et culturelles, inscrites dans une logique d'action collective au niveau local, et qui prennent seulement par la suite le nom de «community arts». L'une des motivations premières des jeunes artistes et comédiens qui en sont à l'origine est d'ouvrir des espaces pour les loisirs et la créativité artistique au sein de quartiers qui en sont dépourvus, dans l'esprit de ce que l'on nomme alors en France l'animation culturelle ${ }^{7}$. Les jeunes membres de Jubilee Theatre and Community Arts Company («Jubilee Arts»), qui s'établissent en 1974 dans la municipalité de Sandwell, limitrophe de Birmingham (East Midlands), conçoivent leur projet en réponse à l'identification d'un manque de structures et d'activités culturelles sur ce territoire :

Sandwell est une vaste municipalité métropolitaine de la région des West Midlands. Elle a une population de 300000 habitants. C'est un territoire très industrialisé composé à l'origine d'un ensemble de petites villes qui ont été regroupées en une municipalité métropolitaine. Elle n'a pas de centre-ville, ne compte aucune organisation d'artistes professionnels et ne dispose que de douze structures de loisirs pour les enfants pour l'ensemble de la municipalité ${ }^{8}$.

6 Leur action consiste à intervenir de manière directe en proposant des activités ludiques, créatives et artistiques à destination de la population. Les enfants sont les premiers concernés par les jeux de groupe, la confection de déguisements en vue de défilés carnavalesques à travers la municipalité, les ateliers d'art plastiques et de théâtre, ou encore la réalisation de grandes fresques murales (murals). Jubilee Arts intervient également auprès de publics adultes en jouant à ses débuts des pièces de théâtre dans la rue ou dans les pubs. A partir de 1978, "the Arts Bus », le bus à impériale de Jubilee Arts reconverti en loge de théâtre, laboratoire photo, atelier d'expression artistique, permet de développer les activités de la compagnie au-delà des activités ludiques : le bus peut faire office d'espace de réunion ou de centre d'accueil et d'information pour des groupes de femmes, de retraités, des associations de locataires. Le bus représente une ressource mobile très efficace qui pallie le manque de lieux dédiés dans la municipalité.

7 On retrouve des principes et modalités d'actions très similaires au sein du groupe InterAction, fondé dans le nord de Londres en 1969 par l'américain Ed Berman (Inter-Action dispose également de son propre bus), dans les activités de Trinity Arts, basé à Small Heath (commune attenante à Birmingham), au Blackfriars Settlement de Southwark (sud de Londres) dont le Photography Project animé par Paul Carter à partir de 1974 forme les participants à la photographie. On pourrait multiplier les exemples de projets à travers le pays : 149 organisations adhèrent à l'Association of Community Artists à sa création en 1974', signe de l'émergence dans le champ culturel britannique d'une tendance forte en faveur du décloisonnement des pratiques artistiques.

8 Ces organisations conçoivent leur action comme des interventions artistiques et culturelles dans le maillage social et associatif parfois fragile des municipalités dans lesquelles elles opèrent. La conscience de profondes inégalités dans l'accès des classes populaires à l'art, entendu non seulement comme l'accès aux œuvres du patrimoine mais aussi comme l'accès aux pratiques artistiques et culturelles, motive leur action. Les acteurs qui s'engagent dans cette démarche sont pour la plupart jeunes, issus de la classe moyenne mais aussi de la classe ouvrière et disposent d'une formation en arts visuels ou arts de la scène. Pour les fondateurs de l'organisation Free Form, dans le nord de Londres, c'est le rejet du conservatisme des écoles d'art et la volonté de produire un art qui se nourrisse de l'interaction avec un public populaire qui les pousse à s'installer dans l'East End de Londres, en transformant un local désaffecté en espace de création ouvert à tous. 
Les artistes Martin Goodrich (Royal College of Art), Jim Ives (Royal Academy) et Barbara Wheeler-Early (Manchester College of Art) fondent Free Form à partir d'un rejet « du monde des galeries d'art pour lequel leur formation les avait préparés mais qui représentait pour eux ce même élitisme qui excluait les publics qu'ils cherchaient à atteindre. ${ }^{10}$ " Cette démarche n'est pas sans évoquer les revendications des étudiants de l'école d'art et de design d'Hornsey, à Londres, occupée en mai 1968 : les étudiants dénoncent la hiérarchie, implicite au sein de l'école, entre beaux-arts et arts appliqués ${ }^{11}$. Ils rejettent également la figure de l'artiste isolé dans son studio, déconnecté des réalités sociales. L'idée de produire un art participatif, présent dans la cité, porteur de significations politiques, est au cœur des revendications. De nombreux étudiants en art s'éloignent à cette époque des milieux culturels institutionnels pour rechercher une pratique artistique qui corresponde à un engagement politique. Andrew Dewdney, membre du collectif Cockpit Arts, dans le quartier de Holborn à Londres témoigne de son expérience :

Nous étions tous profondément influencés par les débats politiques de la période. [...] Au début des années soixante-dix, j'avais conçu une critique politique du monde de l'art et avais conclu que d'un point de vue moral, il valait mieux travailler en dehors des institutions artistiques. [Le Cockpit] essayait de tisser des relations entre l'art contemporain et le monde en dehors des sphères de l'art, c'est-à-dire dans les écoles et auprès de la communauté, en utilisant la photo et la vidéo ${ }^{12}$.

D'autres acteurs du mouvement, notamment au sein de Jubilee Arts, ont une expérience de formation dans le domaine en pleine émergence des Cultural Studies ${ }^{13}$. Ils ont pleinement conscience des constructions idéologiques et sociales qui assurent la prédominance d'une culture des élites, ainsi que des enjeux de classe qui organisent la répartition des ressources dédiées à la culture. La critique des modes d'attribution de financements publics par l'Arts Council est ainsi un élément fondamental dans la structuration des principes du mouvement community arts ${ }^{14}$. Le soutien disproportionné à des grandes institutions nationales, principalement concentrées à Londres et fréquentées par une élite, est critiqué de manière virulente. Ce qui est en cause, ce sont donc les processus d'hégémonie culturelle, c'est-à-dire la valorisation (idéologique, sociale et économique) des formes ou pratiques culturelles dominantes au détriment des possibilités d'expression des groupes sociaux minoritaires. Comme les membres de Trinity Arts en font le constat, la valeur et l'existence même de cultures ouvrières ne sont absolument pas reconnues, ce qui se traduit physiquement dans l'environnement :

Small Heath est une zone urbaine périphérique assez généralement mal entretenue, et qui se trouve aujourd'hui engagée dans un processus de transformation, de rénovation urbaine et d'amélioration. Sa population [...] appartient majoritairement à la classe ouvrière, dont la culture a été pratiquement détruite au sens où très peu d'espaces pour l'expression culturelle subsistent ${ }^{15}$.

10 Les organisations community arts engagent ainsi une réflexion fondamentale sur les cloisonnements qui structurent le champ culturel britannique. Elles agissent de manière pragmatique en pleine conscience des tensions de classes qui opposent culture populaire et culture élitiste. Le développement des pratiques community arts à la fin des années soixante est pleinement contemporain du travail du Centre for Contemporary Cultural Studies à Birmingham : tandis que les chercheurs du Centre portent leur attention sur les transformations sociales, économiques et idéologiques qui affectent la classe ouvrière dans le contexte de l'après-guerre, les artistes impliqués dans la recherche d'une action sociale et culturelle cherchent à donner une voix aux populations minoritaires dont les formes d'expression culturelles sont dévalorisées. L'émergence du mouvement community arts constitue une réponse à la marginalisation des pratiques culturelles populaires. Dans 
son rapport annuel de 1987-1988, au titre évocateur (« Not the Royal Opera ») l'organisation Telford Community Arts redit ce principe: "Nous contestons l'idée que l'art est quelque chose qui existe "pour les autres", pour les "gens aisés", “ceux qui sont cultivés", ou tout autre section privilégiée de la sociétée ${ }^{16}$.»

11 Le mouvement community arts est également redevable de l'antécédent des "arts labs » apparus dans le contexte de la contre-culture des années soixante. Ces lieux de création rassemblant de nombreux médias et disciplines artistiques (graphisme, photo, vidéo, théâtre, performance, cinéma, sérigraphie, posters) apparaissent à Londres à partir de 1967 et se propagent dans les années suivantes dans d'autres villes du pays ${ }^{17}$. Ils se conçoivent comme des espaces hors-institution propices à l'expérimentation artistique, selon des modèles de production alternatifs aux conventions du système des beaux-arts dominant ${ }^{18}$. Les perspectives ouvertes par la contre-culture, notamment la remise en question de l'establishment culturel et le rejet d'une conception descendante de l'art, sont formatrices du contexte critique dans lequel se développe les pratiques community art :

C'est précisément le sentiment de l'épuisement des institutions, des pratiques, des canons et des définitions dans chacune des sphères de la vie culturelle qui a animé une importante partie de l'activité artistique et des dissensions des années soixante 19.

12 Le mouvement représente ainsi un projet de réinvestissement de la culture populaire par le peuple et pour le peuple, comme espace possible d'une résistance collective. Il y a dans cette démarche une critique latente non seulement des processus de domination hégémonique de la culture élitiste, mais aussi des industries culturelles, qui induisent une posture de consommation individuelle des activités de loisirs. Les acteurs des community arts tendent à considérer que la culture authentique se vit en tant que pratique active et créatrice de sens, éminemment collective.

\section{De l'expression artistique à la participation politique : raviver la démocratie à l'échelle de la communauté}

Les projets community art portés au niveau local par les collectifs d'artistes et de «travailleurs culturels ${ }^{20}$ inscrivent leur action dans une démarche de changement social par le bas, selon deux principes fondamentaux: l'accessibilité des moyens de production culturelle et l'encouragement à la participation de chacun ${ }^{21}$. Il s'agit de créer les conditions dans lesquelles les membres d'une communauté disposent des outils de production culturelle et des possibilités d'expression mais sont également en mesure d'exercer un contrôle sur les usages qui sont faits de ces productions culturelles. Organiser l'accès de tous aux pratiques artistiques relève d'une stratégie d'ouverture des expressions de populations marginalisées, devant conduire à des formes d'action sociale collective :

Il ne s'agit pas simplement d'injecter « de l'art dans la communauté », comme un passe-temps éducatif, ou pour faire en sorte que les enfants (et les adultes) ne restent pas dans la rue à ne rien faire. Il existe aujourd'hui de très nombreuses compagnies qui développent des pratiques community art à travers le pays. Dans chacune d'entre elles, des groupes de résidents locaux, des jeunes gens, des locataires, des syndicalistes se tournent vers des modes de communication artistique pour mieux échanger entre eux, pour célébrer leurs intérêts communs, pour explorer le potentiel de ces méthodes en termes d'entraide et de changement social ${ }^{22}$. 
L'objectif est bien de faire entendre la voix de groupes sociaux dominés (économiquement, socialement) et habituellement tenus à l'écart de la participation politique, par l'accès à des formes d'expression créative. Par ce processus, le pari est de faire advenir des solidarités et des convergences et d'insuffler des dynamiques collectives, autour de projets locaux. Le développement des activités d'Inter-Action dans le nord de Londres en fournit un exemple. A partir d'une activité initialement axée sur le théâtre de rue, l'animation d'une troupe d'enfants, et la gestion d'une compagnie ( the Almost Free Theatre »), l'organisation monte des ateliers d'imprimerie, met à disposition des habitants et des associations locales de l'équipement vidéo, audiovisuel ou photographique ("community video", "community media van»), et déploie des activités autour de la lutte contre l'échec scolaire ou l'information pour l'accès aux droits sociaux. La création d'une "ferme urbaine », sur un terrain laissé à l'abandon et réhabilité par les résidents du quartier, illustre la manière dont Inter-Action devient un relais facilitant les initiatives qui émanent des gens eux-mêmes. La ferme, qui compte une écurie et des jardins partagés, est un espace auto-géré, dans lequel les résidents de tous âges s'impliquent sur des tâches diverses :

La ferme a été presque entièrement construite grâce au travail bénévole des habitants du quartier. Des électriciens, des maçons, des charpentiers ont tous donné de leur temps à ce projet, pour servir les intérêts de leur propre communauté. [...] La ferme est la preuve qu'avec un peu d'imagination ce type de projet coopératif peut fonctionner, et ce, sur le long terme ${ }^{23}$.

15 Au-delà de la vocation première d'Inter-Action autour du théâtre, les activités s'élargissent pour prendre en compte les besoins (en termes d'infrastructures de loisir) et les compétences de la population locale, selon un processus qui permet des prises de responsabilité individuelles, au profit du collectif, de la communauté.

Un mot sur un terme de community, dont l'emploi en français est problématique. La richesse sémantique, ainsi que les connotations positives, voire affectives, du terme community n'ont pas d'équivalent en français avec le terme "communauté ». Community renvoie à une échelle de l'organisation sociale essentiellement locale, géographiquement circonscrite. Le terme évoque les dimensions sociales, culturelles et politiques de la vie des habitants d'un quartier, d'une municipalité. Il connote une qualité des relations sociales permises par cette échelle réduite : en raison du caractère direct des relations sociales qu'elle permet, la notion de community apparaît comme plus organique et concrète que society. Elle paraît incarner l'échelle idéale d'une démocratie directe locale. La riche dimension sociale du concept de communauté, imaginée ou réelle, peut dès lors renvoyer à une visée, un objectif: celui de raviver et nourrir le lien social, de faire communauté autour de préoccupations et d'intérêts communs ${ }^{24}$. C'est à ce niveau que les pratiques community arts cherchent à intervenir, comme si quelque chose des relations sociales de proximité était à reconstruire dans des environnements fragilisés par la transformation des sociabilités traditionnelles. L'expression des valeurs et imaginaires collectifs par l'art est envisagée comme l'un des outils de cette démarche.

Profondément influencés par les travaux de théoriciens de l'émancipation tels que Paolo Freire, Augusto Boal ou Ivan Illich, mais aussi par les antécédents du théâtre politique de Joan Littlewood ${ }^{25}$, les projets community arts veulent faire de l'accès à l'expression artistique et culturelle le levier de processus d'autonomisation des individus, devant conduire à des mobilisations politiques ${ }^{26}$. Le concept d'empowerment prend ici tout son sens. Il désigne non seulement le processus par lequel des individus accèdent à une 
conscience politique de leurs conditions matérielles et développent les compétences et la confiance en soi nécessaire pour agir sur ces conditions et les transformer. Il renvoie également au résultat de ce processus: la capacité d'agir des individus ${ }^{27}$. Les organisations community arts se considèrent comme des catalyseurs de dynamiques sociales, devant permettre aux participants de passer de l'accès à l'expression artistique à l'engagement et à la prise de responsabilité dans des actions collectives de portée politique. Il s'agit de donner aux gens les moyens d'être acteurs de leur vie, d'être en situation de contrôle, en commençant par la vie quotidienne à l'échelle locale de la communauté. Maggie Pinhorn, réalisatrice et membre du collectif Tower Hamlets Arts Project créé en 1974 dans ce quartier populaire de Londres, explique la conception qu'elle a de son rôle en ces termes :

Mon travail, c'est de renforcer la confiance en soi des gens, pour faire en sorte qu'ils puissent s'exprimer de manière créative et fassent usage de leur imagination. C'est peut-être l'un des gestes les plus politiques qui soit. [...] Ce dont je me rends compte suite à la participation des gens dans ce genre de projet, ce n'est pas forcément qu'ils vont utiliser le film, la vidéo ou tout autre médium pour s'exprimer, mais c'est le simple fait qu'ils vont oser s'exprimer, dans leur vie personnelle. On peut ainsi travailler sur la confiance qu'une personne a en elle-même afin qu'elle soit en mesure d'aller d'elle-même se plaindre de ses conditions de logement. Qu'elle aille demander un logement décent. Qu'elle aille chercher un boulot, qu'elle réfléchisse et écrive davantage, qu'elle se prenne en main pour améliorer sa qualité de vie ${ }^{28}$.

En termes de principes d'action et d'objectifs, le mouvement community arts est extrêmement proche de l'élan radical incarné par le foisonnement d'initiatives politiques locales, issues de la base, que Peter Hain, militant affilié au Jeunes Libéraux dans les années 1960s et 1970, englobe sous le terme de "community action». Il décrit ce type de mobilisation issue de la base comme «un style d'action politique à travers lequel les gens gagnent la confiance nécessaire pour se mobiliser pour leurs droits et pour la capacité de contrôler leur propre desti ${ }^{29}$.» Le mouvement community arts relève de la même démarche d' empowerment. Le pari est de produire des processus de changement social grassroots, par le biais de la culture et par la libération des imaginaires :

Notre travail, c'est d'essayer de faire en sorte que les gens expriment quelque chose de ce qui touche à la manière dont ils vivent, là où ils vivent, et de les amener finalement à changer les choses là où ils vivent et de prendre le contrôle sur les questions qui les concerne dans leur lieu de vie, à travers l'art. Nous sommes des artistes, et c'est pour cela que l'on travaille avec des moyens artistiques, mais chacun peut le faire de la manière qui lui convient ${ }^{30}$.

Qu'advient-il de ces formes d'engagement par l'art dans le contexte politique et idéologique transformé des années 1980 ?

\section{Les community arts dans les années 1980}

19 L'arrivée au pouvoir du gouvernement conservateur de Margaret Thatcher en 1979 marque un tournant politique et idéologique en Grande-Bretagne. Les effets de ce changement sont perçus de manière directe dans le milieu de la culture, et ont des effets concrets pour le développement et le maintien des pratiques community arts. Les années 1980 sont pour le mouvement le moment d'une réflexion sur le rôle social de l'art, ce que révèle un débat de la période qui oppose les objectifs de démocratisation culturelle et de démocratie culturelle. 
Sur le plan idéologique, le projet politique d'émancipation et de participation au cœur des pratiques community arts ne saurait être plus opposé au nouvel ordre idéologique et axiologique qu'incarne le thatchérisme. Pour la "Nouvelle Droite», ces pratiques représentent les dernières scories de la permissivité des années soixante et les dérives d'un art gauchiste mêlant esthétique et politique. Les discours de Margaret Thatcher sur la culture réaffirment une conception élitiste et hiérarchique de l'art, envisagé comme autonome par rapport au champ politique. On en revient à une définition de la culture qui la réduit à l'ensemble des grandes œuvres de l'esprit qui font le prestige du patrimoine national :

L'art est une dimension vitale de notre civilisation, de notre vision et de notre patrimoine. [...] La santé d'une société dépend autant de sa capacité à mettre à l'écart ce qui est à jeter, que de sa capacité à promouvoir l'excellence ${ }^{31}$.

21 Convoquant l'argument selon lequel l'État se doit de conserver une position de neutralité au nom d'une supposée autonomie de la sphère culturelle, le gouvernement réduit les financements de la culture (en accord avec sa politique générale de réduction des dépenses publiques): pour l'année 1980, le budget alloué à l'Arts Council n'est pas recalculé en tenant compte de l'inflation et se trouve dans les faits considérablement réduit. La baisse des ressources est confirmée dans les années suivantes, palliée dans une certaine mesure par l'arrivée des financements issus du secteur privé. L'institution est contrainte de répercuter les coupes budgétaires sur l'ensemble de ses récipiendaires. Entre les grandes institutions nationales et les petites structures proposant des pratiques artistiques innovantes, l'arbitrage est rapidement fait: en 1981, quarante et un clients, parmi les plus petits et donc les plus dépendants, sont rayés de la liste des récipiendaires de subventions de l'Arts Council ${ }^{32}$.

22 Au cours des années soixante-dix, l'Arts Council avait commencé à prendre en compte les pratiques community arts, en créant diverses commissions d'évaluation et d'attribution de financements spécifiques pour ces nouvelles pratiques atypiques (Community Arts Working Party, Community Arts Committee, Community Arts Evaluation Working Party) ${ }^{33}$. L'Association of Community Artists se constitue en 1974 pour effectuer un travail de lobbying et faire office d'interlocuteur entre l'institution et le réseau naissant de structures community arts. L'ACA prend de l'ampleur au cours des années suivantes: des rencontres annuelles sont l'occasion d'échanges sur les bonnes pratiques, de débats sur les objectifs, de rencontres entre les acteurs issus de différentes régions. L'Association fédère des tendances plus ou moins radicales en son sein. D'une manière générale le mouvement se structure et se définit en grande partie à travers sa relation conflictuelle avec l'Arts Council, dont il critique vertement les choix d'arbitrage en termes de financements ${ }^{34}$. La question du rapport aux financements institutionnels est cependant un sujet qui divise le mouvement. Certaines structures (Telford Arts) se méfient des contraintes associées à l'obtention de subventions, et font le choix de l'autofinancement; d'autres ont recours aux subventions et avancent l'argument selon lequel la promotion de l'accès à l'art relève des obligations de l'Arts Council tels qu'ils sont définis dans sa Charte Royale. Owen Kelly, membre actif de l'ACA et de sa branche plus militante le Shelton Trust, identifie un abandon du radicalisme du mouvement, à partir du moment où les organisations ont été prises dans l'engrenage de l'obtention des subventions, en prise avec les critères d'attribution définis par les financeurs ${ }^{35}$.

Quoiqu'il en soit, le Community Arts Committee, commission dédiée au sein de l'Arts Council, est dissous en 1980 et le processus de délégation de ses compétences aux Regional Arts 
Associations est accéléré ${ }^{36}$. La même année, une nouvelle directive impose aux organisations sollicitant des fonds publics d'avoir le statut de charity, c'est-à-dire d'association caritative à but non lucratif. L'une des restrictions inhérentes à ce statut est l'impossibilité de poursuivre des objectifs politiques, en raison d'un impératif de neutralité. L'ACA fait le choix d'endosser ce statut, laissant à ses adhérents la possibilité de continuer à occuper l'espace militant. C'est la mission dont est investi le Shelton Trust, créé en $1979^{37}$. Le groupe diffuse Another Standard, magazine qui reflète une tendance radicale au sein du mouvement, et publie un manifeste en 1986 intitulé Culture and Democracy: the Manifesto ${ }^{38}$. Ce texte est un appel urgent lancé aux milieux militants pour mettre la culture au centre d'un projet de transformation sociale qui ne se cantonne pas seulement aux questions politiques et économiques. Le manifeste représente une tentative de fédérer le mouvement qui s'est donné jusqu'alors peu de textes théoriques, et semble davantage tourné vers la pratique que vers la théorisation de ses propres positions. C'est à cette période que paraissent les premiers ouvrages qui tentent de définir l'histoire, les principes et les objectifs des pratiques community arts $^{39}$.

Le thème central du manifeste est la démocratie culturelle, qui permet de mettre en regard les principes du mouvement community arts et ceux de l'Arts Council. La notion d'accessibilité, dans l'acception de ce dernier, repose sur l'idée que les efforts de l'institution doivent se porter sur le processus d'une "démocratisation de la culture ", entendue comme la culture légitime, à diffuser aux masses. Il s'agit de faire connaître au plus grand nombre les œuvres d'un canon artistique établi. Dans le cadre des pratiques community arts, en revanche, la notion d'accessibilité renvoie à la mise à disposition des outils de l'expression à l'ensemble de la population ainsi qu'à l'égale reconnaissance de la diversité de ces expressions :

La notion de démocratie culturelle s'articule autour des notions de pluralité et d'égalité d'accès aux moyens de production et de distribution culturelle. Elle suppose que la production culturelle est ancrée dans le contexte plus large des discours sociaux. [...] La démocratisation culturelle, à l'inverse, peut être décrite comme le fait d'imposer obligatoirement, à l'ensemble de la société, les seules valeurs d'un groupe particulièrement puissant. Ces valeurs apparaissent comme neutres et naturelles. [...] Le mouvement community arts doit faire de l'idée de la démocratie culturelle son objectif principal, et en accord avec cet objectif, il doit exiger la décentralisation des moyens de production culturelle ${ }^{40}$.

Les années 1980 sont une période complexe pour le mouvement. Elles sont marquées par la poursuite de débats fondamentaux sur la culture et le changement social. On observe une volonté de recentrage sur les principes radicaux, notamment autour du Shelton Trust, alors que l'engagement des organisations dans les mobilisations politiques de la décennie se poursuit : l'antiracisme, les droits des femmes, la défense des droits sociaux, le soutien aux mobilisations des chômeurs ainsi qu'aux luttes syndicales sont autant de sujets sur lesquels les organisations community arts se mobilisent. Jubilee Arts met ainsi ses ressources et compétences au service de mobilisations telles que la marche des chômeurs en 1981 («The People's March for Jobs » qui part de Liverpool pour rejoindre Londres), et ses membres sont présents auprès des mineurs de Kellingley au long de la grève des mineurs de $1984-1985^{41}$. Cependant un essoufflement de l'élan radical initial se fait également sentir. De nombreuses organisations luttent pour survivre économiquement. La tendance pragmatique consistant à accepter d'édulcorer un propos politique semble avoir raison des objectifs de transformation sociale radicale à l'origine du mouvement. Un autre facteur de dépolitisation est l'évolution des pratiques community arts vers les milieux culturels institutionnels et leur incorporation dans les politiques culturelles municipales ${ }^{42}$ 
. On peut aussi lire dans cette évolution une reconnaissance dans les milieux culturels et sociaux de la validité de principes défendus par le mouvement tels que l'accessibilité et la participation des groupes sociaux marginalisés à la production de contenus culturels.

\section{Conclusion}

Les pratiques community arts se développent à partir de la fin des années soixante dans le contexte d'une remise en question des conceptions traditionnelles de la culture. Elles se caractérisent par une démarche militante qui associe à la critique des logiques de domination culturelle un discours de transformation sociale et culturelle radicale. Le mouvement s'inscrit dans un faisceau de mobilisations grassroots qui militent pour une transformation démocratique de la société, selon le principe que le changement social doit provenir de la base, en rejet des processus de domination. Les acteurs engagés dans ses pratiques mettent en lumière la marginalisation des expressions culturelles populaires et contribuent activement aux débats critiques sur la nécessité d'une reconnaissance de la diversité culturelle.

Le mouvement contribue de manière essentielle à la redéfinition du concept de culture, vers une compréhension beaucoup plus anthropologique du terme. La culture y est requalifiée et étirée vers la notion d'expression, qui permet d'aller au-delà des constructions hiérarchiques opposant professionnel et amateur, expert et profane, noble et populaire. Ce changement de perspective introduit de manière cruciale la dimension de classe dans les théories de la culture et permet de mettre en évidence les processus d'hégémonie culturelle. Les passerelles entre le mouvement et les perspectives ouvertes à la même époque par les Cultural Studies apparaissent clairement.

Au cours des années 1980, il semble que sur le plan idéologique la lutte devienne d'autant plus difficile que les thèmes de la droite conservatrice s'imposent: individualisme et célébration de l'entreprise privée, remise en question de l'action collective, retour d'une conception classique de la culture qui justifie un désengagement de l'État dans le domaine des politiques culturelles. Comme l'analyse François Matarasso, spécialiste de l'histoire de ce mouvement, le terme community art est progressivement éclipsé dans les années 1990 par celui de " participatory art » : une transition qui signale une dépolitisation par rapport à la visée de changement social et à la rhétorique de l'action collective des années 1970 :

La trajectoire allant de "community art" à "participatory art", si elle a été vue comme une mesure pragmatique par ceux qui y ont œuvré, signala et accompagna une transition depuis les modes d'action politisés et collectifs des années 1970 vers le type de programmes artistiques dépolitisés et centrés sur les individus, que finance aujourd'hui l'argent public au Royaume-Uni. ${ }^{43}$.

D'une manière générale, alors que les organisations community arts sont contraintes à adopter une posture défensive dans les années 1980, le radicalisme du mouvement perd de sa force et de sa cohérence. C'est aussi le signe de l'acceptation des pratiques de la démocratie participative dans la gouvernance locale: les acteurs «historiques » font aujourd'hui le constat d'une prise en compte bien plus systématique par les autorités locales des problématiques d'inclusion des membres des communautés dans les politiques publiques locales qui les concernent ${ }^{44}$. Les pratiques de démocratie participative, défendues par le mouvement community arts, se sont institutionnalisées.

Mathilde Bertrand est Maîtresse de Conférences en Civilisation Britannique à l'Université Bordeaux-Montaigne (Laboratoire CLIMAS, EA 4196). Ses travaux 
portent sur la culture visuelle britannique, notamment dans le contexte des mobilisations politiques de la seconde moitié du $\mathrm{XX}^{\mathrm{e}}$ siècle. Elle est l'auteur d'une thèse sur l'histoire de la photographie indépendante en Grande-Bretagne de la fin des années cinquante à la fin des années quatre-vingt.

\section{BIBLIOGRAPHIE}

ANOTHER STANDARD, Culture and Democracy: The Manifesto, Londres, Comedia, 1986.

BACQUÉ Marie-Hélène et BIEWENER Carole, L'empowerment, une pratique émancipatrice, Paris, La Découverte, 2013.

BOAL Augusto, Theatre of the Oppressed, Londres, Pluto Press, 1979.

BRADBY David et CAPON Susanna (dir.), Freedom's Pioneer : John McGrath's Work in Theatre, Film and Television, Exeter, University of Exeter Press, 2005.

BRADEN Su, Artists and People, Londres, Routledge and Kegan Paul, 1978.

CREHAN Kate, Community Art, An Anthropological Perspective, Oxford, New York, Berg, 2011.

DEWDNEY Andrew, entretien avec Shirley Read, 3 avril 2000, Oral History of British Photography, National Sound Archive, British Library. C459/114.

FITZGERALD Sandy (dir.), An Outburst of Frankness. Community Arts in Northern Ireland. A Reader, Dublin, Tasc at New Island, 2004.

FREE FORM ARTS TRUST Ltd, Cultural Action for Community Development : A Report on the Goldsmith's Square Estate, London, Paris, unESCO, février 1985.

FREIRE Paulo, Pédagogie des opprimés ; Conscientisation et révolution, Paris, Maspero, 1974 (1e édition en anglais en 1970).

GREED Clara, Planning in the UK : An Introduction, Hondmills and New York, Palgrave Macmillan, 2014.

HAIN Peter, Community Politics, Londres, J. Calder, 1976.

HAYNES Jim, « An Alternative Marriage ? », International Times 66, 10-13 octobre 1969.

HIGGINS Lee, Community Music: In Theory and In Practice, Oxford, Oxford University Press, 2012.

HUTCHINSON Robert, The Politics of the Arts Council, London, Sinclair Browne, 1982.

ILLICH Ivan, Tools for Conviviality, London, Calder \& Boyars, 1973.

ILlICH Ivan, Deschooling Society, Harmondsworth, Penguin, 1976.

INTER-ACTION archives, « Investing in Leisure. Out of the Waste Land », Municipal and Public Services Journal, 12 septembre 1975, Archives d'Inter-Action conservées dans les archives de l'Arts Council, Blythe House, Londres.

JUBILEE ARTS, Annual Report 1979-80, West Bromwich, 1980. 
JUBILEE ARTS ARCHIVE, « 1977 », film footage, c. 1977, https://vimeo.com/120796300.

KELLY Owen, Community, Art and the State: Storming the Citadel, London, Comedia, 1984.

KING Sylvia, « Making Mansions », in KENNEDY Liam (dir.), Remaking Birmingham : The Visual Culture of Urban Regeneration, Oxon, London, New York, Routledge, 2004.

MATARASSO François, « "All in this Together” : The Depolitisation of Community Art in Britain, 1970-2011 ", in van ERVEN Eugene (dir.), Community Art Power, Rotterdam, International Community Arts Festival (ICAF), 2013, p. 214-240.

MOORE-GILBERT Bart et SEED John (dir.), Cultural Revolution? The Challenge of the Arts in the 1960s, London, Routledge, 1992.

MORIARTY Gerry et JEFFERS Alison, " "Where have we come from?” Community Arts to Contemporary Practice », Community Arts Unwrapped, https:// communityartsunwrapped.com/2014/03/24/where-have-we-come-from-community-arts-tocontemporary-practice/.

NEVEU Erik, Sociologie des mouvements sociaux, Paris, La Découverte, 2015.

NEVILLE Richard, Playpower, Exploring the International Underground, Londres, Cape, 1970.

NIGG Heinz et WADE Graham, Community Media, Community Communication in the United Kingdom : Video, Local TV, Film and Photography, Zurich, Regenbogen-Verlag, 1980.

SINCLAIR Andrew, Arts and Cultures; The History of the Fifty Years of the Arts Council of Great-Britain, Londres, Sinclair-Stevenson, 1995.

TELFORD COMMUNITY ARTS, Not the Royal Opera, c.1988, Annual Report 1987-1988, Arts Council Archive, London, Blythe House.

THATCHER Margaret, « Speech at the Royal Academy Banquet », 22 mars 1980. http:// www.margaretthatcher.org/document/104370

TICKNER Lisa, Hornsey 1968 : The Art School Revolution, Londres, Frances Lincoln, 2008.

TOURAINE Alain, La Voix et le Regard, Paris, Seuil, 1978.

TRINITY ARTS, « GASP and Friends - application for financial support April 76-March 77 », 1976. Archive papers, Arts Council of Great Britain, Blythe House.

WILLIAMS Raymond, Keywords, A Vocabulary of Culture and Society, Londres, Fontana, [1976] 1983. YounG Michael et WILMOTT Peter, Family and Kinship in East London, Harmondsworth, Penguin, [1957], 1971.

\section{NOTES}

1. "Community arts is moved by a political interest in putting the means of artistic production into the hands of the people ", Graham Peete, entretien avec l'auteur à West Bromwich, 3 juin 2010.

2. TOURAINE, Alain, La Voix et le Regard, Paris, Seuil, 1978.

3. NEVEU Erik, Sociologie des mouvements sociaux, Paris, La Découverte, 2015, p. 11.

4. FREIRE Paulo, Pédagogie des opprimés; Conscientisation et révolution, Paris, Maspero, 1974 ; ILLICH Ivan, Tools for Conviviality, London, Calder \& Boyars, 1973; ILLICH Ivan, Deschooling Society, 
Harmondsworth, Penguin, 1976; BOAL Augusto, Theatre of the Oppressed, Londres, Pluto Press, 1979.

5. GREED Clara, Planning in the UK: An Introduction, Hondmills and New York, Palgrave Macmillan, 2014, p. 115.

6. young Michael et wiLмотт Peter, Family and Kinship in East London, Harmondsworth, Penguin, [1957], 1971, p. 142.

7. L'animation culturelle et la figure de l'animateur dans les quartiers populaires en France sont des références constantes dans le discours des acteurs des community arts. Entretien avec Graham Peete.

8. «Sandwell is a large metropolitan borough in the West Midlands. 300,000 people live in it. It's a very heavily industrialised area and used to be a lot of small towns which have now been formed into a large metropolitan borough. It doesn't have a city centre, the borough has no professional arts group and as far as provision for play for children goes, it has only twelve play centres to service the entire borough. " JUBILEE ARTS ARCHIVE, « 1977 », film footage, c. 1977, mis en ligne au printemps 2015, https://vimeo.com/120796300, consulté le 3 mars 2017.

9. Kelly Owen, Community, Art and the State: Storming the Citadel, London, Comedia, 1984, p. 13.

10. «The Free Form project began as rejection. [...] These artists wanted to escape the gallery world for which their training had prepared them but which to them embodied the very elitism that excluded those they wanted to reach. » CREHAN Kate, Community Art, An Anthropological Perspective, Oxford, New York, Berg, 2011, p. 4.

11. TUCKNER Lisa, Hornsey 1968 : The Art School Revolution, Londres, Frances Lincoln, 2008.

12. "We were all infected by the politics of the period. [...] By the early seventies, [I] had developed a political critique of the art world and decided that the moral high ground was in working outside the institutions of art. [The Cockpit] was trying to make these connections between contemporary art, and the world outside of the art world, in the schools and the community, using photo and video. » DEWDNEY Andrew, entretien avec Shirley Read, 3 avril 2000, Oral History of British Photography, National Sound Archive, British Library. C459/114.

13. Stephen Lacey, membre de Jubilee Arts, a suivi une formation au Centre for Contemporary Cultural Studies, dirigé par Stuart Hall à partir de 1968 au sein de l'université de Birmingham. MORIARTY Gerry et JEFFERS Alison, " "Where have we come from?" Community Arts to Contemporary Practice ", Community Arts Unwrapped, mis en ligne le 24 mars 2014, https:// communityartsunwrapped.com/2014/03/24/where-have-we-come-from-community-arts-tocontemporary-practice/, consulté le 3 mars 2017.

14. Kelly Owen, Community, Art and the State, op. cit.; fitzGerald Sandy (dir.), An Outburst of Frankness. Community Arts in Northern Ireland. A Reader, Dublin, Tasc at New Island, 2004.

15. "Small Heath is a generally run-down inner-ring area in the midst of redevelopment, renewal and general improvement. The population [...] is predominantly working class, the culture of which has been almost totally suppressed in that opportunities for cultural expression rarely exist. » TRINITY ARTS, « GASP and Friends - application for financial support April 76-March $77 », 1976$. Archive papers, Arts Council of Great Britain, Blythe House.

16. "We challenge the notion that the arts are something for "other people" to do, for the "welloff", for the "well-educated", or other privileged sections of society. » TELFORD COMMUNITY ARTS, Not the Royal Opera, c.1988, Annual Report 1987-1988, Arts Council Archive, London, Blythe House, p. 2.

17. L'Arts Lab de Birmingham, par son dynamisme et sa longévité, joue un rôle particulièrement important en tant qu'espace artistique alternatif et pépinière de nombreuses initiatives culturelles et artistiques dans la région des West Midlands. Voir KING Sylvia, « Making Mansions ", in KENNEDY Liam (dir.), Remaking Birmingham: The Visual Culture of Urban Regeneration, Oxon, London, New York, Routledge, 2004, p. 55. 
18. MOORE-GILBERT Bart et SEED John (dir.), Cultural Revolution? The Challenge of the Arts in the 1960s, London, Routledge, 1992 ; HAYNES Jim, « An Alternative Marriage? », International Times 66, 10-13 octobre 1969, p. 13 ; NEVILLE Richard, Playpower, Exploring the International Underground, Londres, Cape, 1970.

19. «It was precisely a sense of the exhausted potential of central institutions, practices, canons and definitions in sphere after sphere of cultural life that animated much of the activity and dissension of the 1960s. " MOORE-GILBERT et SEED, Cultural Revolution ?, op. cit., p. 9.

20. Nombreux sont les membres des organisations étudiées qui rejettent les connotations élitistes, voire romantiques que comporte le terme "artiste " et qui préfèrent être considérés comme des «travailleurs culturels» (cultural workers), voire, comme le souligne Sylvia King (de Jubilee Arts) comme des «militants culturels» (cultural activists). Voir KING, « Making Mansions », op. cit, p. 55.

21. Entretien avec Graham Peete; NIGG Heinz et WADE Graham, Community Media, Community Communication in the United Kingdom : Video, Local TV, Film and Photography, Zurich, RegenbogenVerlag, 1980.

22. "It is not just "art in the community", an educational hobby, or simply a way of keeping kids (and adults) off the streets. There are now hundreds of community arts companies up and down the country. In each of them, groups of local residents, young people, tenants, and trades unionists are using the arts to communicate more effectively with each other, to celebrate their own interests, and to explore the potential for self-help and change. " JUBILEE ARTS, Annual Report 1979-80, West Bromwich, 1980, p. 5.

23. "The farm was almost entirely built by volunteer work from the local community. Electricians, builders, carpenters, all gave their time to the scheme in the interest of their own neighbourhood. [...] The farm is proving that with a bit of imagination this sort of self-help sheme can be made to work and to continue to work. » INTER-ACTION archives, "Investing in Leisure. Out of the Waste Land », Municipal and Public Services Journal, 12 septembre 1975, Archives d'Inter-Action conservées dans les archives de l'Arts Council, Blythe House, Londres.

24. WILLIAMS Raymond, Keywords, A Vocabulary of Culture and Society, Londres, Fontana, [1976] 1983, p. 75-76.

25. Dans les années cinquante, Joan Littlewood et sa compagnie Theatre Workshop font vivre un théâtre politique, qui s'adresse à la classe ouvrière et puise sa matière dans la réalité sociale. Suivant cet héritage, John Fox, fondateur de la compagnie Welfare State International en 1968, ou John McGrath et Elizabeth MacLennan, qui fondent en 1971 la compagnie 7:84, cherchent des " alternatives radicales aux pratiques culturelles établies ». BRADBY David et CAPON Susanna (dir.), Freedom's Pioneer : John McGrath's Work in Theatre, Film and Television, Exeter, University of Exeter Press, 2005, p. xix. Ces objectifs sont très proches de ceux des community artists.

26. «Early community arts practitioners were very conscious of drawing on radical arts history and of using this history both as inspiration and practical source of information. We did not suffer from collective amnesia. » MORIARTY et JEFFERS, « "Where have we come from?" Community Arts to Contemporary Practice ", op. cit.

27. BACQUÉ Marie-Hélène et BIEWENER Carole, L'empowerment, une pratique émancipatrice, Paris, La Découverte, 2013.

28. «I am in the business of building up people's confidence in order that they can express themselves creatively and use their imaginations. That is possibly the most political act that you can be doing. [...] What I can see as a result of people having been involved in that kind of work is that they are not going to do it in terms of film or video or anything else, but in their personal lives. So you might build up somebody's confidence enough for them to complain about their housing conditions. To go along and demand a decent flat to live in. To get a job, to think and to 
write more, to do whatever else for themselves to improve their own quality of life. » NIGG et WADE, Community Media, Community Communication in the United Kingdom, op. cit., p. 182.

29. "A style of political action through which people gain the confidence to agitate for their rights and the ability to control their own destinies. » HAIN Peter, Community Politics, Londres, J. Calder, 1976.

30. "What we're trying to do is to get the people to express something about the way they live, about their own area, in fact to make changes in their area and take control of some aspects within their own area through the arts. We're artists so we do it through the arts but anyone can do it in any area. " JUBILEE ARTS ARCHIVE, op.cit.

31. «Art] is a vital part of our civilisation, of our vision, and our heritage. [...] The health of society depends as much on the discouragement of rubbish as on the fostering of excellence. » THATCHER Margaret, «Speech at the Royal Academy Banquet», 22 mars 1980. http:// www.margaretthatcher.org/document/104370, consulté le 3 mars 2017.

32. SINCLAIR Andrew, Arts and Cultures; The History of the Fifty Years of the Arts Council of Great-Britain , Londres, Sinclair-Stevenson, 1995, chapitre 10.

33. En 1972, alors que l'Arts Council dispose de 9,3 millions de livres de dotation, il finance cinquante-sept organisations et artistes définis comme relevant de la catégorie Community Arts pour un total de 176000 livres, et, l'année suivante, 350000 livres sont attribués à 75 projets : NIGG et WADE, Community Media, Community Communication in the United Kingdom, op. cit., p. 30 ; KELLY Community, Art and the State, op. cit., p. 15. Les subventions s'élèvent à un million de livres en 1978: SINCLAIR, Arts and Cultures, op. cit., p. 224. S'il y a une certaine reconnaissance par l'Arts Council de l'existence d'un mouvement community arts, le soutien institutionnel reste très marginal. La révolution voulue par la tendance plus radicale de l'ACA n'a pas lieu.

34. KING, « Making Mansions », op. cit, p. 57, 59.

35. KELLY Community, Art and the State, op. cit., chapitre 4.

36. Ces structures régionales composées d'élus, d'acteurs de terrain et d'associations deviennent l'interlocuteur principal des organisations community arts pour leurs demandes de subvention. Les politiques culturelles locales ainsi que les dotations des Regional Arts Associations ne sont pas homogènes sur le territoire, et certaines comme West Midlands Arts, Northern Arts, et Greater London Arts Association sont plus ambitieuses dans leur soutien aux pratiques community arts. Voir HUTCHINSON Robert, The Politics of the Arts Council, London, Sinclair Browne, 1982. p.168.

37. HIGGins Lee, Community Music: In Theory and In Practice, Oxford, Oxford University Press, 2012, p. 34 .

38. ANOTHER STANDARD, Culture and Democracy: The Manifesto, Londres, Comedia, 1986.

39. BRADEN Su, Artists and People, Londres, Routledge and Kegan Paul, 1978; Kelly Owen, Community, Art and the State, op. cit.; NIGG et WADE, Community Media, Community Communication in the United Kingdom, op. cit.

40. "Cultural democracy revolves around the notion of plurality, and around equality of access to the means of cultural production and distribution. It assumes that cultural production happens within the context of wider social discourses [...] The democratisation of culture can be seen as the compulsory imposition, on society at large, of the values of one particularly powerful group. These values appear as neutral, and as natural. [...] The community arts movement must take the idea of cultural democracy as its overall aim, and with regard to that aim, it must clearly, and loudly, demand the decentralisation of the means of cultural production. » KELLY Owen, Community, Art and the State, op. cit., p. 101.

41. Entretien avec Brendan Jackson, membre de Jubilee Arts, juin 2010.

42. KING, « Making Mansions », op. cit.

43. "The path from "community art" to "participatory art", whilst seen as merely pragmatic by those who made it, marked and allowed a transition from the politicised and collectivist action of 
the seventies, towards the depoliticised, individual-focused arts programmes supported by public funds in Britain today." MATARAsso François, «"All in this Together" : The Depolitisation of Community Art in Britain, 1970-2011", in van Erven Eugene (dir.), Community Art Power, Rotterdam, International Community Arts Festival (ICAF), 2013, p. 216.

44. FREE FORM ARTS TRUST Ltd, Cultural Action for Community Development : A Report on the Goldsmith's Square Estate, London, Paris, UNESCO, février 1985, p. 2.

\section{RÉSUMÉS}

Le mouvement community arts apparaît en Grande-Bretagne à la fin des années soixante, dans le sillage de la contre-culture. Portées par des artistes issus de différentes disciplines artistiques, les pratiques community arts visent à ouvrir l'accès à la création artistique aux groupes sociaux défavorisés. Diverses et foisonnantes, ces pratiques prennent la forme d'un mouvement engagé dans une redéfinition du rôle social de l'art, et pour l'avènement d'une "démocratie culturelle » (Shelton Trust, Culture and Democracy: The Manifesto, 1986). Dans les formulations que se donne le mouvement dans les années soixante-dix et quatre-vingt, cette revendication est indissociable d'un discours politique radical qui a pour objectif l'émancipation des individus et le décloisonnement des conceptions traditionnelles de la culture. A l'époque où les Cultural Studies développent une critique des hiérarchisations idéologiques qui opposent culture noble et culture populaire, le mouvement community art envisage la culture populaire comme le site d'une résistance possible aux formes de domination idéologique.

Analyser le mouvement community arts à travers le prisme de la notion d'engagement nous invite à interroger les mobilisations des acteurs et les modalités d'implication des populations locales. A un autre niveau, il apparaît que les revendications portées par les pratiques community arts dépassent largement le seul domaine artistique et contribue au développement d'une théorie de la culture éminemment politique: comment les concepts d'expression populaire et $d$ ' empowerment ont-ils été articulés au sein de ces pratiques? Si le mouvement se définit au cours des années soixante-dix selon une éthique de l'action collective, qu'advient-il de son élan radical dans le contexte idéologique de la décennie suivante, marquée par l'ascendance de valeurs individualistes et conservatrices?

The community arts movement appeared in the United-Kindgom in the wake of the counterculture of the 1960s. Inspired by artists from different disciplines, community arts practices aimed at giving access to artistic creativity to deprived social groups. These multifarious practices formed a movement committed to a redefinition of the social role of art and to the advent of a genuine "cultural democracy" (Shelton Trust, Culture and Democracy: The Manifesto, $1 \mathrm{k986}$ ). In the formulations that the movement developed in the 1970s and 1980s, this demand was inseparable from a radical political discourse of empowerment and challenge to traditional conceptions of culture. At a time when Cultural Studies were developing a critique of ideological hierarchies opposing high and low culture, the community art movement invested popular culture as a possible site of resistance against ideological domination.

To study the community arts movement through the prism of the concept of commitment is an invitation to question the involvement of actors and the modes of implication of local populations in projects. At another level, the challenges raised by community arts practices went well beyond the artistic field and contributed to a political theory of culture: how did community 
arts combine the concepts of cultural expression and empowerment? Whereas the movement defined itself in terms of an ethics of collective action in the 1970s, what happened to this initial radicalism in the ideological context of the 1980s, characterised by the rise of individualistic and conservative values?

INDEX

Mots-clés : art, communauté, engagement, empowerment, militantisme culturel

Keywords : art, community, commitment, activism, empowerment

\section{AUTEUR}

MATHILDE BERTRAND

Laboratoire CLIMAS (Cultures et Littératures des Mondes Anglophones), EA 4196, Université Bordeaux-Montaigne 